\title{
Retrograde intrarenal surgery (RIRS)
}

\author{
Andreas J Gross, Christopher Netsch \\ Department of Urology, Asklepios Hospital Barmbek, Rübenkamp Hamburg, \\ Germany.
}

\section{Key words: flexible renoscopy, renal stones, lasertripsy \\ Introduction}

Technological progress of ureterorenoscopes (URS) and endoscopic instrumentation has evolved retrograde intrarenal surgery (RIRS) into a safe and efficacious modality for the treatment of the upper urinary tract. These improvements in technology have expanded the potential indications of RIRS to intrarenal large stones $(>25 \mathrm{~mm})$, SWL failure, infundibular stenosis, morbid obesity, renoureteral malformations, musculoscelettal deformities and bleeding diathesis [1-7]. In selected patients, RIRS can be considered even for multiple intrarenal calculi with a high stone-free rate and low morbidity. However, neither EAU nor AUA guidelines recommend RIRS as the treatment of choice for intrarenal stones so far. [1, 2,8,9,10].

\section{Instrumentation}

\section{Guidewire}

The placement of a safety wire facilitates and maintains access to the upper urinary tract, although successful wireless RIRS has been currently shown [11]. Ideally, a guidewire requires little force to flex in response to resistance encountered along a tortuous path, while contrarily requiring a large force to perforate tissue. In an in vitro examination, the lubricous, softtip nitinol Glidewire ${ }^{\mathrm{TM}}$ (Boston Scientific Corp., Natick, MA) demonstrated to be the safest wire for initial access to the ureter, as it is less

Correspondence :

Gross AJ,

Fax.+49401818 829829

Email : an.gross@asklepios.com likely to perforate and more likely to bend when a point of obstruction is encountered [12]. Hybrid wires, such as the Sensor ${ }^{\mathrm{TM}}$ (Boston Scientific) and UroWIRE XFTM (Applied Medical, Rancho Santa Margarita, CA) have been developed to combine various advantageous features: a smooth, hydrophilic distal tip, a kink-resistant nitinol body, and a flexible proximal tip. The clinical application of these wires is limited by being more expensive than standard wires. However, it is possible that a hybrid wire may decrease the need to open a second guidewire when a standard wire fails to pass a point of obstruction [13].

\section{Ureteral access sheath}

Ureteral access sheaths facilitate the insertion and straight alignment of the URS into the upper urinary tract during multiple stone fragment extractions, decrease operative time and cost, minimize patient morbidity, and optimize overall success with RIRS [14]. The access sheath also allows efflux of irrigant fluid through the sheath and around the URS, maintaining intrapelvic pressures below $20 \mathrm{~cm} \mathrm{H} 2 \mathrm{O}$ with irrigant fluid pressurized up to $200 \mathrm{~cm} \mathrm{H} 2 \mathrm{O}$ [15]. However, the reliance on axial dilating force of current ureteral access sheaths exposes the urothelium to the potential risk of an abrasive shearing force. A new balloon-based ureteral access sheath combines radial balloon dilation and access sheath placement in a single step and may reduce both the axial force and urothelial disruption [16].

\section{Ureteral balloon dilator/catheters}

Ureteral balloon dilation may be utilized in approximately $5 \%$ of cases when the ureteral access sheath will not advance to the site of pathology due to ureteral stricture, spasm, or a tight ureteral orifice [13]. 


\section{Irrigation}

Adequate irrigation flow is necessary to maintain visibility. Flexible URS have either two separate channels (one working channel for an instrument and the second for irrigation) or one common channel for both irrigation and working instruments. Irrigation can be provided by gravity, a pressure bag, or a variety of hand or foot pumps. It should be emphasized that manual hand irrigation has been shown to produce pressures greater than $100 \mathrm{mmHg}$ in the kidney [17], which may lead to bacteraemia, sepsis, due to increased fluid absorption.

\section{Flexible ureteroscope}

Advancements in flexible URS design and functionality have led to improved lower pole calyceal access and instrument longevity. Ureteroscopes differ in terms of working channel, optical resolution, accessing all calyces, and the durability of the scopes. The newer, actively deflecting flexible URS offer increased lower pole access compared to the older passively deflecting scopes [13]. It was demonstrated that the ideal outer diameter of a flexible ureteroscope, defined in terms of the ease of introduction without the need for ureteral dilation, was 7.4F [18]. Another critical consideration regards the durability of flexible URS: Traxer et al. demonstrated deterioration of maximal dorsal/ventral deflection from $270^{\circ}$ to $133^{\circ}$ and $270^{\circ}$ to $208^{\circ}$ after 50 consecutive RIRS with the same scope (Flex-X, K. Storz, Tuttlingen, Germany) [19]. It was suggested that scopes that return from major repairs have less than $25 \%$ of the life expectancy of a new scope, and it may be more cost effective to replace than repair [20].

\section{Intracorporeal lithotriptors}

Intracorporeal lithotripsy is used for large stone fragmentation. Holmium(Ho):YAG laser lithotripsy has been shown to fragment all compositions of urinary calculi, as well as produce smaller stone fragments than pneumatic or electrohydraulic lithotripsy [21,22]. In addition, the Ho:YAG laser energy is absorbed efficiently in a fluid medium, minimizing the risk of urothelial injury compared to the electrohydraulic lithotrite. Furthermore, retropulsion of the stone is less likely than with a pneumatic lithotrite. However, using the Ho:YAG laser with a deflected flexible URS elevates the risks for collateral damage to instrumentation, including the flexible URS [23].

\section{Stone retrieval devices}

Since Dormia presented a spiral basket for stone retrieval [24], basket design has improved with regard to wires (number, material, shape), shaft (size, material), and configuration (spherical, helical, paired wire, tipless) $[25,26]$. Previously used stainless steel gave way to the soft 'memory metal' Nitinol ${ }^{\circledR}$, a flexible and kink-resistant alloy of nickel and titanium, which is also less rigid than stainless steel and imposes less limitation on URS deflection [25]. Important properties of these devices include visibility during stone manipulation, sufficient radial force to open in the ureter, and the ability to capture, retain, or if necessary disengage a stone. Tipless nitinol-based basket designs are more versatile and atraumatic in stone retrieval due to the unique pliability of the wires and the flexibility to allow full lower pole deflection of a flexible URS in the majority of cases, when compared to alligator or rat tooth forceps $[27,28]$.

\section{The RIRS procedure Preparation of the patient}

Preparation of the patient includes informed consent and explanation of the potential complications and the possibility of insertion of an ureteral stent [29]. General anaesthesia is favoured, but neuroleptic anaesthesia is also feasible.

\section{Antibiotic prophylaxis}

The rate of urinary tract infection following ureteroscopy ranges from 4 to $25 \%$ even when prophylactic antibiotics are administered. Therefore, the use of preoperative antibiotic prophylaxis is still controversial [30]. Patients at risk with preoperative ureteral stent, catheter or nephrostomy tube should be treated with antibiotic prophylaxis as well as patients with heart valves and murmurs at risk for bacterial endocarditis, immunocompromised patients, and drug or radiation induced immunosuppression [30,31 ].

\section{Positioning of the patient}

Ureteroscopy has been described in supine, prone, and 
flank position. The flank position has only been demonstrated with flexible instruments []. In the author's institution patients are positioned routinely in the dorsal lithotomy position.

\section{Cystoscopy}

Flexible or rigid cystoscopes can be used for initial guide wire placement into the ureter, depending on urologist's training and availability of the equipment. Cystoscopes and URS should be combined with a video monitoring system as it is ergonomically beneficial, ideal for teaching trainees, and for documentation. At the author's institution, rigid cystoscopy is the preferred modality, although they may cause more ureteral trauma, particularly in males [29].

\section{Safety guide wire}

Fluoroscopy is used to confirm the position of the guide wire as it is advanced. The wire acts as a safety wire. A general principle of RIRS is always to have a safety wire present intraoperatively.

\section{Ureteroscope insertion}

When a safety wire is in place, a second working wire must be inserted in order to backload the endoscope, allowing the wire to act as a guide for the instrument to be advanced into the ureter or kidney under fluoroscopic guidance [33]. Placement of the second wire can be achieved by the cystoscope or the placement of an ureteral access sheath over the first wire. The bladder should be emptied before removing the cystoscope to facilitate insertion of the flexible URS. Otherwise, cannulation of the ureteric orifice with the URS might be hindered.

\section{Ureteral stenting following ureteroscopy}

Placement of an ureteral catheter or internal ureteral stent has been the standard of care following RIRS to prevent renal colic by obstructing stone fragments or ureteral edema after surgery [29]. However, routine stenting might add morbidity to the procedure by infection, dysuria, flank pain, haematuria or stent migration. In a randomised controlled trial, patients assigned to ureteral stents following ureteroscopy had increased pain compared with non-stented patients, yet the incidence of emergency room visits, urosepsis, and hospitalization and stone free rates did not differ between the two groups [34,35]. They concluded, not to stent the ureter routinely if the stone burden is small, the ureter has not been balloon dilated and the Ho:YAG laser has been used without ureteral trauma [35]. Although these series analyzed the usefulness of stenting after ureteral stone extraction, these principles can also be applied on RIRS. Strict indications for stent insertions are: ureteral perforation, ureteral dilation greater than $10 \mathrm{~F}$, ureteral edema due to stone treatment/size, failure to advance the RIRS procedure due to narrow ureter or ureteral orifice, infected urinary system with an obstructing system, large stone burden with many fragments remaining to pass, and solitary kidney [29].

\section{Postoperative care}

RIRS is typically performed as an outpatient day procedure. If a stent is left indwelling it is typically removed 3 to 10 days after the procedure. Postoperative radiographs are obtained within 1 to 2 weeks to determine success of the procedure [29]. Small stone fragments $(<4 \mathrm{~mm})$ normally pass after the stent has been removed due to the passive ureteral dilation that occurs from stenting [29,37]. Postoperative imaging has been recommended to determine residual calculi or silent obstruction $[29$,$] .$ Silent obstruction may occur secondarily from ureteral edema, trauma, stricture, and result in renal failure if it remains undetected. However, other working groups do not recommend routine imaging if there is no history of pre-existing ureteral stricture, perforation, or significant stone impaction at time of surgery [38].

\section{RIRS for the treatment of kidney stones}

RIRS has progressed to be an alternative to SWL and PCNL for treating renal calculi offering the low morbidity of SWL combined with stone-free rates comparable with PCNL for small to moderate-sized renal calculi [39]. Significant lower pole stone burden is the limiting factor for success. Even with new flexible URS the lower pole calyces can only be accessed in $93 \%$ of cases [5]. Fabrizio et al. reported a $77 \%(50 \%)$ stone-free rate for renal stones $<10 \mathrm{~mm}$ $(>16 \mathrm{~mm})$ with a complication rate of $3 \%$ [40]. For single intrarenal stones $>20 \mathrm{~mm}$ [4], and $30 \mathrm{~mm} \mathrm{[2],}$ the stone free rates were $93.3 \%$ and $90.9 \%$ after an 
average of 2.4 and 1.8 procedures, respectively. There are a few published series regarding RIRS for multiple unilateral intrarenal stones [5, 7, 9, 10, 40,41]. Breda et al. reported overall stone-free rates after one and two procedures of $64.7 \%$ and $92.2 \%$ for multiple unilateral intrarenal stones and a complication rate of $13.6 \%$ [9]. Herrera et al. presented the largest series on RIRS for multiple renal stones so far. They reported an overall stone-free rate after one procedure of $74.4 \%$ for multiple unilateral intrarenal stones and a complication rate of $5.6 \%$, respectively [10]. To conclude, this series supports the usefulness of RIRS throughout the renal collecting system with a high success and low complication rate $[9,10,40,42-45]$.

\section{Ureteroscopy in children}

Since Shepherd et al. [46] and Ritchey et al. [47] have published their experience, ureteroscopy has gained widespread acceptance among paediatric urologists. The safety and efficacy of ureteroscopy in children has been confirmed [48-52], although these series differ in terms of instruments, techniques, and the location of calculi. The access to the upper urinary tract remains controversial due to the risk of ureteral trauma and/or bleeding. Shepherd et al. have shown that dilation of the ureter up to $12 \mathrm{~F}$ did not result in the development of vesicoureteral reflux (VUR) postoperatively [46]. In addition, VUR resolved spontaneously with conservative management in most children []. Some authors believe that stent preplacement provides a safe and effective alternative in achieving access to the child's ureter resulting in higher overall stone-free rates, as shown in adults [54]. Antegrade ureteral access may be considered in children who have a percutaneous nephrostomy in place and a completely obstructed ureter [53, 55]. In addition, ureteroscopic disintegration of ureteral calculi has gained acceptance [48-53]. The outcome in prepubertal children were comparable with those in adults [56]. Nerli et al. currently reported an overall stone free rate after one RIRS procedure (with Ho:YAG lithotripsy) of $90 \%$ for ureteral calculi [52]. They concluded that complete stone clearance after one procedure is possible if the stone is small $(<10 \mathrm{~mm})$, solitary, and below the level of the pelviureteral junction [52].

\section{Complications of RIRS Urosepsis}

Manipulation of the stone in presence of an infection is likely to result in bacteraemia and possible urosepsis. In patients who present with an infected collected system and an obstructing stone, urinary decompression should be the initial treatment followed by culture sensitive antibiotic treatment. Postoperative bacteraemia and sepsis should be treated with culture sensitive intravenous antibiotics followed by oral antibiotics when the patient has defervesced [29].

\section{Ureteral avulsion}

Ureteral avulsion is an uncommon but serious complication of ureteroscopy. Avulsion typically occurs during basket extraction of a stone fragment that is too large and becomes caught in the ureteral mucosa, telescoping and avulsing the ureter as the stone is extracted. To avoid these catastrophic complication, a safety wire should be always used. And, the stones should be broken into small enough fragments for ureteral passage [29].

\section{Ureteral stricture}

The ureteral perforation rate has decreased concurrently with the ureteral stricture rate over the past 20 years ranging from 0 to $5.9 \%$. Risk factors for ureteral perforation included operation time, stone location, use of electrohydraulic lithotripsy, and surgeon`s experience [29].

\section{References}

1. Preminger GM, Tiselius HG, Assimos DG, et al. Guideline for the management of ureteral calculi. J Urol 2007;178:2418-34.

2. Riley JM, Stearman L, Troxel S. Retrograde ureteroscopy for renal stones larger than $2.5 \mathrm{~cm}$. J Endourol 2009; 23:1395-8

3. Yinghao S, Yang B, Gao X. The management of renal caliceal calculi with a newly designed ureteroscope: a rigid ureteroscope with a deflectable tip. JEndourol 2010; 24:23-6.

4. Breda A, Ogunyemi O, Leppert JT, et al. Flexible ureteroscopy and laser lithotripsy for single intrarenal 
stones $2 \mathrm{~cm}$ or greater-is this the new frontier? J Urol. 2008; 179:981-4.

5. Grasso M. Ureteropyeloscopic treatment of ureteral and intrarenal calculi. Urol Clin North Am 2000; 27:623-31.

6. Grasso M, Bagley D. In discussion of: Small diameter, actively deflectable, flexible ureteropyeloscopy. J Urol 1998; 160:1648-53.

7. Wen CC, Nakada SY. Treatment selection and outcomes: renal calculi. Urol Clin North Am 2007; 34:409-19.

8. Tiselius HG, Ackermann D, Alken $\mathrm{P}$, et al. Guidelines on urolithiasis. Eur Urol 2001; 40:362-71.

9. Breda A, Ogunyemi O, Leppert JT, et al. Flexible ureteroscopy and laser lithotripsy for multiple unilateral intrarenal stones. Eur Urol 2009;55:1190-7. 10. Herrera-Gonzalez G, Netsch C, Oberhagemann K, et al. Effectiveness of single flexible ureteroscopy for multiple renal calculi. J Endourol. 2011; 25:431-5.

11. Dickstein RJ, Kreshover JE, Babayan RK, et al. Is a safety wire necessary during routine flexible ureteroscopy? J Endourol. 2010; 24:1589-92.

12. Clayman M, Uribe CA, Eichel L, et al. Comparison of guide wires in urology. Which, when and why? J Urol 2004; 171:2146-2150.

13. Holden T, Pedro RN, Hendlin K, et al. Evidencebased instrumentation for flexible ureteroscopy: a review. J Endourol. 2008; 22:1423-6.

14. Kourambas J, Byme RR, Preminger GM. Does a ureteral access sheath facilitate ureteroscopy? J Urol 2001;165:789-793.

15. Rehman J, Monga $\mathrm{M}$, Landman $\mathrm{J}$, et al. Characterization of intrapelvic pressure during ureteropyeloscopy with ureteral access sheaths. Urology 2003; 61:713-718.

16. Harper JD, Ebrahimi KY, Auge BK, et al. Comparison of a novel radially dilating balloon ureteral access sheath to a conventional sheath in the porcine model. J Urol. 2008; 179:2042-5.

17. Auge BK, Pietrow PK, Lallas CD, et al. Ureteral access sheath provides protection against elevated renal pressures during routine flexible ureteroscopic stone manipulation. J Endourol. 2004; 18:33-6.

18. Hudson RG, Conlin M, Bagley D. Ureteric access with flexible ureteroscopes: Effect of the size of the ureteroscope. BJU Int 2005; 95:1043-1044.

19. Traxer O, Dubosq F, Jamali K, et al. New- generation flexible ureterorenoscopes are more durable than previous ones. Urology 2006; 68:276279.

20. Carey RI, Gomez CS, Maurici G, et al. Frequency of ureteroscope damage seen at a tertiary care center. J Urol 2006; 176:607-610.

21. Chan KF, Vassar GJ, Pfefer TJ, et al. Holmium:YAG laser lithotripsy: A dominant photothermal ablative mechanism with chemical decomposition of urinary calculi. Lasers Surg Med 1999; 25:22-37.

22. Teichman JM, Vassar GJ, Bishoff JT, et al. Holmium: YAG lithotripsy yields smaller fragments than lithoclast, pulsed dye laser or electrohydraulic lithotripsy. J Urol 1998; 159:17-23.

23. Vassar GJ, Teichman JM, Glickman RD. Holmium:YAG lithotripsy efficiency varies with energy density. J Urol 1998; 160:471-476.

24. Dormia E. Dormia basket: Standard technique, observations, and general concepts. Urology 1982; 20: 437.

25. Zeltser IS, Bagley DH. Basket design as a factor in retention and release of calculi in vitro. J Endourol. 2007; 21:337-342.

26. Monga M, Hendlin K, Lee C, et al. Systematic evaluation of stone basket dimensions. Urology 2004; 63: 1042-1044.

27. Bagley D, Ramsay K, Zeltser I. An update on ureteroscopic instrumentation for the treatment of urolithiasis. Curr Opin Urol 2004; 14:99-106.

28. Honey RJ. Assessment of a new tipless nitinol stone basket and comparison with an existing flat-wire basket. JEndourol 1998; 12:529-531.

29. Chew BH, Denstedt JD. Ureteroscopy and Retrograde Ureteral Access. In Campbell-Walsh eds, Urology, 9th edn, Vol.II. Chapt 45 Philadelphia: Saunders, 2007: 1508-1525.

30. Grabe M. Controversies in antibiotic prophylaxis in urology. Int J Antimicrob Agents. 2004; 23 Suppl 1:S17-23.

31. Amin M. Antibacterial prophylaxis in urology: a review. Am J Med. 1992; 92:114S-117S.

32. Herrell SD, Buchanan MG. Flank position ureterorenoscopy: new positional approach to aid in retrograde caliceal stone treatment. J Endourol. 2002; $16: 15-8$.

33. Afane JS, Olweny EO, Bercowsky E et al. Flexible 
ureteroscopes: a single center evaluation of the durability and function of the new endoscopes smaller than 9Fr. J Urol. 2000; 164:1164-8.

34. Borboroglu PG, Amling CL, Schenkman NS et al.Ureteral stenting after ureteroscopy for distal ureteral calculi: a multi-institutional prospective randomized controlled study assessing pain, outcomes and complications. J Urol. 2001; 166:16517.

35. Denstedt JD, Wollin TA, Sofer M et al. A prospective randomized controlled trial comparing nonstented versus stented ureteroscopic lithotripsy. J Urol. 2001;165:1419-22.

36. Deliveliotis C, Giannakopoulos S, Louras G et al. A. Double-pigtail stents for distal ureteral calculi: an alternative form of definitive treatment. Urol Int. 1996; 57:224-6.

37. Harmon WJ, Sershon PD, Blute ML, et al.Ureteroscopy: current practice and long-term complications. J Urol. 1997; 157:28-32

38. Bugg CE Jr, El-Galley R, Kenney PJ, et al. Followup functional radiographic studies are not mandatory for all patients after ureteroscopy. Urology. 2002; 59:662-7.

39. Galvin DJ, Pearle MS. The contemporary management of renal and ureteric calculi. BJU Int 2006; 98:1283-8.

40. Fabrizio MD, Behari A, Bagley DH. Ureteroscopicmanagement of intrarenal calculi. J Urol 1998; 159:1139-43.

41. Preminger GM, Assimos DG, Lingeman JE, et al. Chapter 1:AUA guideline on management of staghorn calculi: diagnosis and treatment recommendations. J Urol 2005; 173:1991-2000.

42. Gupta PK. Is the holmium:YAG laser the best intracorporeal lithotripter for the ureter? A 3-year retrospective study. J Endourol 2007; 21:305-9.

43. Grasso M, Beaghler M, Loisides P. The case for primary endoscopic management of upper urinary tract calculi: II. Cost and outcome assessment of 112 primary ureteral calculi. Urology 1995; 45:372-6.

44. Grasso M. Experience with the holmium laser as an endoscopic lithotrite. Urology 1996;48:199-206.

45. Stav K, Cooper A, Zisman A, et al. Retrograde intrarenal lithotripsy outcome after failure of shock wave lithotripsy. J Urol 2003; 170:2198-201.

46. Shepherd P, Thomas R, Harmon EP. Urolithiasis in children: Innovations in management. J Urol 1988; 140:790-792.

47. Ritchey M, Patterson DE, Kelalis PP, et al. A case of pediatric ureteroscopic lasertripsy. J Urol 1988; 139:1272-1274.

48. Koura AC, Ravish IR, Amarkhed S, et al. Ureteroscopic stone management in prepubertal children. Pediatr Surg Int. 2007; 23:1123-1126

49. Smaldone M, Cannon GM Jr, Wu HY, et al. Is ureteroscopy first line treatment for pediatric stone disease? J Urol 2007; 178:2128-2131.

50. Satar N, Zeren S, Bayazit Y, et al. Rigid ureteroscopy for the treatment of ureteral calculi in children. J Urol 2004; 172:298-300.

51. Tan AH, Al-Omar M, Denstedt JD, et al. Ureteroscopy for pediatric urolithiasis: An evolving first-line therapy. Urology 2005; 65:153-156.

52. Nerli RB, Patil SM, Guntaka AK, et al. Flexible ureteroscopy for upper ureteral calculi in children. $\mathrm{J}$ Endourol. 2011; 25:579-82.

53. Reddy PP. Pediatric ureteroscopy. Urol Clin North Am 2004; 31:145-156.

54. Rubenstein RA, Zhao LC, Loeb $\mathrm{S}$, et al. Prestenting improves ureteroscopic stone-free rates. J Endourol 2007; 21:1277-1280.

55. Gupta R, Manohar T, Desai MR. Antegrade flexible ureteroscopy in supine position for impacted multiple ureteric calculi. Ind J Urol 2006; 22:139-141. 56. Reddy PP, Nishinaka K, DeFoor W. Ureteroscopy is safe and effective in prepubertal children. $\mathrm{J}$ Endourol 2003; 17:A212. 